\title{
Frequency modulation detection as a measure of temporal processing: Age-related monaural and binaural effects
}

\author{
John H. Grose and Sara K. Mamo \\ Dept. Otolaryngology - Head \& Neck Surgery, University of North Carolina at Chapel Hill, Chapel \\ Hill, NC 27519, USA \\ John H. Grose: jhg@med.unc.edu
}

\begin{abstract}
The detection of low-rate frequency modulation (FM) carried by a low-frequency tone has been employed as a means of assessing the fidelity of temporal fine structure coding. Detection of lowrate FM can be made more acute, relative to the monaural case, by the addition of a pure tone to the contralateral ear. This study examined whether FM detection in the $500-\mathrm{Hz}$ region could be further improved by using a binaural stimulation mode where the modulator was antiphasic across the two ears. The study also sought to determine whether these dichotic FM conditions were beneficial in identifying the emergence of a temporal fine structure processing deficiency relatively early in the aging process. Young, mid-aged, and older listeners ( $\mathrm{n}=12$ per group) were tested. The results demonstrated better FM acuity in the dichotic task irrespective of listener age. Dichotic FM detection also differentiated between age groups more definitively than diotic detection, especially in terms of distinguishing mid-aged from older listeners. In the group of older listeners, dichotic FM detection was weakly associated with absolute sensitivity to the carrier. In addition, this group failed to show a dichotic benefit in the presence of a marked asymmetry in sensation level across ears. The overall pattern of results suggests that dichotic FM measurements have advantages over monaural measurements for the purposes of assessing age-related temporal processing effects, although a marked asymmetry in absolute thresholds across ears could undermine these advantages.
\end{abstract}

\section{Keywords}

frequency modulation detection; age; temporal processing; monaural; binaural

\section{Introduction}

Psychophysical assessment of temporal fine structure processing in the auditory system requires a task that can be interpreted in terms of the strength of underlying neural synchrony, or phase locking. One task that has been used in this context is the detection of frequency modulation (FM) at low carrier and modulation frequencies (Lacher-Fougere and Demany, 1998; Moore and Sek, 1996). The notion is that the ability to track the

\footnotetext{
(C) 2012 Elsevier B.V. All rights reserved.

Corresponding Author: John H. Grose, Dept. OHNS, UNC-CH, G190 Physicians Office Building, CB\#7070, 170 Manning Drive, Chapel Hill, NC 27599-7070, Voice: (919) 966-3342, Fax: (919) 966-7941, jhg@ med.unc.edu.

Publisher's Disclaimer: This is a PDF file of an unedited manuscript that has been accepted for publication. As a service to our customers we are providing this early version of the manuscript. The manuscript will undergo copyediting, typesetting, and review of the resulting proof before it is published in its final citable form. Please note that during the production process errors may be discovered which could affect the content, and all legal disclaimers that apply to the journal pertain.
} 
instantaneous frequency of a slowly modulated tone relies more on the temporal pattern (phase locking) of the neural response than on place cues in ears with good phase locking. This task has been used to gauge fine structure coding in focus populations such as listeners with cochlear hearing loss (Buss et al., 2004; Ernst and Moore, 2012; Moore and Skrodzka, 2002; Strelcyk and Dau, 2009), and older listeners (He et al., 2007). In all of these studies, FM detection was measured using monaural presentation. Binaural tasks have also been used to assess the processing of temporal fine structure. Many of these have involved some measure of sensitivity to interaural time/phase differences as a function of frequency (Grose and Mamo, 2010; Hopkins and Moore, 2010; Lacher-Fougere and Demany, 2005; Moore et al., 2012a).

In terms of gauging the strength of phase locking, it is reasonable to suggest that binaural measures may offer a more sensitive indicator of neural synchrony than monaural measures. The basis for this is that, for measures that reflect the comparison of phase-locked inputs from the two ears, deficits at the binaural level will be greater than deficits at the monaural level. This has been clearly demonstrated byBatra et al. (1997), who showed that the synchronization index of cells in the superior olive to binaural inputs was the product of the synchronization indices to the respective monaural inputs - the monaural indices typically being less than 1.0. Somewhat tempering this viewpoint is the finding ofMoore et al. (2012b) who found only a moderate correlation between the results of monaural and binaural psychophysical tests designed to measure sensitivity to temporal fine structure, suggesting that the two tests were measuring partly different abilities. Nevertheless, the argument that binaural measures of fine structure coding might be more sensitive than monaural measures raises the question of whether the sensitivity of the FM detection task can be increased by employing it in a binaural context. Evidence supporting this comes from a study byWitton et al. (2000), who showed that the detection threshold for low-rate FM could be an order of magnitude more acute if a steady tone was presented to the ear contralateral to that receiving the modulated tone. The dichotic advantage was present for FM rates up to about $40-60 \mathrm{~Hz}$, and occurred even for interaural level differences of 40-50 $\mathrm{dB}$. The dichotic stimulus configuration used byWitton et al. (2000), as well as in earlier studies (e.g. Green et al., 1976), results in sinusoidal interaural phase modulation. One purpose of the present study was to determine whether an advantage could be gleaned from using a stimulus configuration that also resulted in dynamic interaural phase modulation, but in which the two ears received antiphasic FM (rather than one ear receiving a steady tone). This configuration effectively doubles the depth of interaural phase modulation.

The interest in binaural FM detection in this study occurs within the context of the effects of advancing age on temporal processing. There is converging psychophysical and electrophysiological evidence that the coding of temporal fine structure declines with age, and independently of hearing loss (Clinard et al., 2010; Grose and Mamo, 2010; Moore et al., 2012a; Ross et al., 2007). This has led to an interest in the development of tests of temporal fine structure processing that can be applied in the clinic (e.g., Hopkins and Moore, 2010; 2011). In addition, there is an interest in the time course of this decline, with a focus on the emergence of temporal processing deficits relatively early in the aging process (Dobreva et al., 2011; Grose and Mamo, 2010; Moore et al., 2012b). The purpose of this study therefore was twofold: (1) to determine whether the dichotic FM configuration tested here is a sensitive measure of temporal processing, particularly with respect to its monaural counterpart; and (2) to determine whether this measure identifies a pre-senescent emergence of temporal fine structure processing deficiency. The corresponding hypotheses were that: (1) dichotic FM detection is more sensitive than monaural FM detection for normal-hearing listeners irrespective of listener age; and (2) older listeners show less benefit from dichotic presentation than younger listeners, and that this effect is evident in middle-age. 


\section{Method}

\subsection{Participants}

A cohort of 36 listeners participated, 12 in each of three age groups: Younger (19-29 yrs, 7 female), Mid-Aged (43-57 yrs, 6 female), and Older (65-77 yrs, 9 female). All listeners had normal audiometric thresholds ( $\leq 20 \mathrm{~dB} H L$ ) across the octave frequency range $250-4000$ $\mathrm{Hz}$, except for three Older listeners whose thresholds at $4000 \mathrm{~Hz}$ ranged from $25-35 \mathrm{~dB}$ $\mathrm{HL}$ in at least one ear. There was no interaural threshold asymmetry, with the mean absolute interaural difference across frequencies and listeners being about $4 \mathrm{~dB}$. The averaged audiograms for the three age groups are shown in Fig. 1.

\subsection{Stimuli}

All stimuli in the FM detection task were $1250 \mathrm{~ms}$ in duration, including 25-ms raised cosine rise/fall ramps. The FM waveform was given by:

$$
\mathrm{x}(\mathrm{t})=\mathrm{A} \sin \left(2 \pi \mathrm{f}_{\mathrm{c}} \mathrm{t}+\beta \sin \left(2 \pi \mathrm{f}_{\mathrm{m}} \mathrm{t}\right)\right)
$$

where $\mathrm{A}$ is the amplitude of the signal, $\mathrm{s}$ is the modulation index (i.e., 'FM depth'), $\mathrm{f}_{\mathrm{c}}$ is the carrier frequency, and $\mathrm{f}_{\mathrm{m}}$ is the modulation frequency. For all conditions, $\mathrm{f}_{\mathrm{m}}=2 \mathrm{~Hz}$, yielding 2.5 cycles of modulation per stimulus. The starting phase of the modulator was always 0 radians for monaural and diotic presentations, and 0 and $\pi$ radians when the modulator was out of phase at the two ears. The carrier frequency was randomly roved on a presentation-by-presentation basis across the range $460-540 \mathrm{~Hz}$, with random starting phase. The purpose of the frequency rove was to undermine the ability to use place cues to detect FM since the listener could not establish a reliable 'place anchor' against which to compare frequency shifts within the observation intervals of a forced-choice trial. Stimuli were generated for each presentation at a sampling rate of $25 \mathrm{kHz}$, and presented at $65 \mathrm{~dB}$ SPL through Etymotic Research ER2 insert phones. There were five presentation conditions: (1) monaural left [Monaural-L]; (2) monaural right [Monaural-R]; (3) binaural presentation of identical tones [Diotic]; (4) dichotic presentation wherein a pure tone was presented to the left ear and an FM tone to the right ear [Dichotic PT/FM]; (5) dichotic presentation wherein an FM tone was presented to each ear but the modulator phase was inverted between ears [Dichotic FM/FM].

In order to obtain a more fine-grained measure of auditory sensitivity at the frequency region of interest to this study, absolute thresholds for a $500-\mathrm{Hz}$ pure tone were also measured. The stimulus for this measurement was $400 \mathrm{~ms}$ in duration, including $25-\mathrm{ms}$ raised cosine rise/fall ramps.

\subsection{Procedure}

FM detection thresholds were measured using a three-alternative forced-choice (3AFC) procedure that incorporated a three-down, one-up stepping rule to converge on the $79.4 \%$ correct point. In the two standard intervals, the stimulus was an unmodulated pure tone; in the signal interval (chosen at random), the stimulus was an FM tone. The inter-stimulus interval was $500 \mathrm{~ms}$. The intervals were marked by lights on a response box, and the listener was instructed to select the interval that was different by means of a button press; correctinterval feedback was provided visually following each trial. Following three correct responses in a row, the depth of FM was reduced; following one incorrect response, the depth was increased. The FM depth was initially changed by a factor of $\sqrt{2}$ and, following two reversals in direction of depth change, the factor was further reduced by its square root. A track continued for 10 reversals, and the mean of the final six reversal depths was taken as 
the threshold estimate for that track. At least three estimates of threshold were collected for each condition, and the final threshold value was taken as the mean of all estimates. All listeners were allowed to practice on each of the five conditions until performance across repeated runs appeared stable. Averaged across listeners within an age group, the younger group received 1.6 practice runs per condition, the mid-aged group received 1.9 practice runs per condition, and the older group received 3.1 practice runs per condition prior to data retention. Once practiced, the runs were blocked by condition but the order of conditions was varied across listeners.

The same 3AFC procedure was used to adaptively measure absolute threshold for a $500-\mathrm{Hz}$ tone. Observation intervals were $400 \mathrm{~ms}$ in duration with an inter-stimulus interval of 400 ms. The step size for tone level was initially $8 \mathrm{~dB}$, and this was halved after the second and fourth reversals in level direction. The step size remained at $2 \mathrm{~dB}$ for the final 6 reversals, and the threshold estimate was taken as the mean signal level at the final 6 reversal points. Three estimates of threshold were obtained for each ear, with a fourth obtained if the range of the initial three exceeded $3 \mathrm{~dB}$. The final threshold value was taken as the mean of all estimates. The 500-Hz tone thresholds were collected prior to the FM thresholds.

\section{Results}

\subsection{0-Hz pure-tone thresholds}

As a preliminary step, the pure-tone thresholds at $500 \mathrm{~Hz}$ were inspected. A repeated measures analysis of variance (ANOVA) with one within-subjects factor (ear) and one between-subjects factor (age group) indicated no effect of ear $(F(1,33)=0.624 ; p=0.435)$ and no interaction between ear and age group $(\mathrm{F}(2,33)=1.702 ; \mathrm{p}=0.198)$. However, the effect of age group was significant $(F(2,33)=7.839 ; p=0.002)$. Post-hoc testing using Tukey HSD indicated that the younger group had significantly lower thresholds than the older group, but the threshold differences between the younger and mid-aged group, and between the mid-aged group and the older group, did not reach significance. The average $500-\mathrm{Hz}$ threshold collapsed across ears was 6.3, 10.8, and 13.7 dB SPL for the younger, mid-aged, and older groups, respectively. Thus, despite the general classification of clinically 'normal hearing' at $500 \mathrm{~Hz}$ for all listeners, the younger group had lower thresholds than the older age group. The data also indicated that there were no meaningful asymmetries between ears; across all listeners, the average difference between left and right ear thresholds was $0.5 \mathrm{~dB}$, with an average absolute difference of $3 \mathrm{~dB}$. The hearing sensitivity of these listeners ensured that the 65-dB SPL stimuli in the FM detection task was associated with an average SL of least $50 \mathrm{~dB}$ for all three groups.

\subsection{FM detection thresholds}

Evaluation of the FM detection thresholds began with an assessment of two aspects of the data: (1) the symmetry of monaural acuity across ears; and (2) the benefit of listening with two ears in the absence of interaural differences (diotic stimulation). Although monaural FM detection thresholds were measured separately for the left and right ears, there was no $a$ priori reason to expect these to differ. This was confirmed with a paired-samples test that indicated no difference between the two monaural conditions $(t(35)=0.473 ; p=0.639)$. Accordingly, the monaural FM thresholds were collapsed across ears for each listener to yield a single measure of monaural FM acuity. These data are shown as bars in Fig. 2 along with the diotic data. The bar shading indicates age group, as shown by the key, and the error bars show 1 standard deviation. Also shown in Fig. 2 as separate symbols are data from other studies that measured monaural low-rate FM detection for listeners with normal hearing (Buss et al., 2004; He et al., 2007; Strelcyk and Dau, 2009; Witton et al., 2000). Data fromHe et al. (2007) for 5-Hz FM detection are shown separately for younger (black 
triangle) and older (gray triangle) listeners. Although procedural differences exist across the various studies, it can be seen that the general level of performance observed here is in line with previous reports, and that the age effect reported byHe et al. (2007) appears to be present - as expanded on below.

The question of whether detecting FM with two ears is better than with one was addressed by comparing the monaural and diotic thresholds by means of a repeated measures ANOVA with one within-subjects factor (presentation mode) and one between-subjects factor (age group). It should be noted that all ANOVAs on the FM detection data were performed on the $\log$ transforms of the data (cf Buss et al., 2004; Strelcyk and Dau, 2009). There was a significant effect of presentation mode $(F(1,33)=105.26 ; p<0.001)$ and age group $(F(1,33)$ $=12.01 ; \mathrm{p}<0.001)$, but no interaction between these factors. This pattern of results indicates that, for all listeners, diotic detection of FM was superior to monaural detection. ${ }^{1}$ In terms of the age effect, post-hoc analysis using the Tukey HSD test indicated that the younger group had lower detection thresholds than both the mid-aged and older groups $(\mathrm{p}=$ $0.013 \& \mathrm{p}<0.001$, respectively), but that the detection thresholds of the mid-aged and older groups did not differ from each other $(\mathrm{p}=0.173)$.

The main interest of this study was in determining the relative benefit of interaural difference cues for FM detection. For reference, the diotic detection thresholds were used rather than the monaural thresholds - because they represent the greater acuity for FM in the absence of interaural differences. The data for the three binaural conditions (Diotic, Dichotic $P T / F M$, and Dichotic FM/FM) are shown in Fig 3. Each cluster of bars corresponds to a condition as shown on the abscissa, and bar shading indicates age group as shown in the key. Error bars show +1 standard deviation. Also shown as filled squares are data fromWitton et al. (2000) for $\mathrm{f}_{\mathrm{m}}=2 \mathrm{~Hz}$. Although diotic performance in the present data set was similar to the monaural performance of their three experienced listeners, their Dichotic $P T / F M$ thresholds were markedly lower than those measured here. It is not entirely clear what accounts for this difference, but methodological differences across studies could have contributed. Comparing the present study toWitton et al. (2000), these differences include presentation level (65 dB SPL vs. $80 \mathrm{~dB}$ SPL), stimulus duration (1250 ms vs. $1500 \mathrm{~ms})$, and threshold definition (79.4 vs. $75 \%$ correct). To determine the effects of diotic vs. dichotic presentation in the present data, a repeated-measures ANOVA was undertaken with one within-subjects factor (condition) and one between-subjects factor (age group). There were significant effects of condition $(\mathrm{F}(2,66)=103.2 ; \mathrm{p}<0.001)$ and age group $(\mathrm{F}(2,33)=$ $20.5 ; \mathrm{p}<0.001)$. The interaction between these two factors was also significant $(\mathrm{F}(4,66)=$ $3.9 ; \mathrm{p}=0.007)$. Post-hoc testing with Tukey HSD indicated that the three age groups all differed significantly from each other. This pattern of results suggests that the significant interaction between age group and condition reflects a relative disparity in the benefit of transitioning from a diotic to a dichotic listening mode across age groups. This suggestion is supported by the observation in Fig. 3 that the older group, in addition to performing more poorly overall, also appeared to show less benefit from the dichotic cues. To assess this, an analysis was undertaken on the proportional change in FM detection thresholds in the two dichotic conditions relative to the diotic condition. This manipulation essentially normalizes each listener's performance with respect to his/her diotic threshold. Again, the repeated measures ANOVA had one within-subjects factor (condition) and one between-subjects factor (age group). There was a significant effect of condition $(F(1,33)=20.8 ; p<0.001)$ and

\footnotetext{
${ }^{1}$ Although tangential to the focus of this study, the relationship between monaural and diotic performance in general has been a topic of considerable interest. For example, at sufficiently high masker levels the monaural signal-in-noise threshold (NmSm) is equivalent to the diotic threshold (NoSo), but this is not the case at very low masker levels. The parsimonious point of view is that when signal detection is dominated by internal noise that is largely independent at the two ears, diotic performance will be superior to monaural performance. For further discussion, see Langhans and Kohlrausch (1992).
} 
age group $(\mathrm{F}(2,33)=6.3 ; \mathrm{p}=0.005)$, but no interaction between these two factors $(\mathrm{F}(2,33)=$ $1.71 ; \mathrm{p}=0.197)$. Post-hoc testing with Tukey HSD indicated that the proportional improvement in FM detection thresholds did not differ between the younger and mid-aged groups, but that both of these groups showed greater proportional improvement than the older group. Pairwise comparisons indicated that this pattern of results held for both dichotic conditions. In other words, the proportional improvement in acuity for FM detection in dichotic conditions relative to the diotic reference was significantly less for the older listeners than for the younger and mid-aged groups; the latter two groups did not differ in their relative improvement. Older listeners therefore do not benefit from dichotic presentation to the same extent as younger and mid-aged listeners.

Although in proportional terms the mid-aged group showed the same amount of benefit for dichotic listening as did the younger group, their performance was generally poorer than for the younger group. Pair-wise comparisons indicated that the mid-aged group had higher FM detection thresholds than the younger group for the Diotic and Dichotic FM/FM conditions $(\mathrm{p}=0.007$ and 0.033 , respectively), with a similar but non-significant trend for the Dichotic $P T / F M$ condition $(\mathrm{p}=0.082)$.

One issue that must be considered in interpreting these age-related effects is whether the differences in FM detection acuity across age groups are related to differences in absolute thresholds. Recall that the $500-\mathrm{Hz}$ pure-tone thresholds for the younger group were slightly but significantly lower than for the older group. Although one approach to assessing this type of issue is by means of an analysis of covariance (ANCOVA), this approach assumes homogeneity of regression slopes wherein the relationship between the dependent variable (here, FM detection threshold) and the covariate (here, 500-Hz pure-tone threshold) must be constant across all levels of the independent variable (here, age group). To test this assumption, the Dichotic FM/FM thresholds and the 500-Hz pure-tone thresholds for the three different age groups were submitted to a generalized linear model. The Dichotic FM/ $F M$ threshold was the dependent variable in this analysis because it yielded the best binaural listening performance for all groups. The $500-\mathrm{Hz}$ pure-tone threshold was entered as a continuous variable, and listener group was entered as a categorical variable. The main effect of age group was significant (Wald $X^{2}=7.32, \mathrm{p}=0.026$ ), but the main effect of 500$\mathrm{Hz}$ pure-tone threshold was not (Wald $X^{2}=2.10, \mathrm{p}=0.147$ ). There was a significant interaction between age group and 500-Hz pure-tone threshold (Wald $X^{2}=10.192, \mathrm{p}=$ 0.006 ), indicating a significant difference across age groups in the relationship between the 500-Hz pure-tone threshold and the Dichotic FM/FM threshold. In other words, the assumption of homogeneity of regression slope was not met. Inspection of the data for linear trends revealed a positive correlation between the $500-\mathrm{Hz}$ pure-tone threshold and the Dichotic FM/FM threshold for the older adults, but not for the younger or mid-aged subjects. A one-tailed Pearson's correlation test confirmed the significant association between the two thresholds for the older age group $(r(10)=0.553, p<0.05)$. This pattern of results indicates that, although acuity to dichotic FM was related to absolute threshold for the carrier frequency in the older listeners, this association was not apparent in other age groups. Thus, the differences in FM detection acuity across age groups are unlikely to be due to differences in absolute thresholds, especially as the presentation level was on average at least $50 \mathrm{~dB}$ SL.

\section{Discussion}

This study was designed to test two hypotheses: (1) dichotic FM detection is better than monaural FM detection for normal-hearing listeners irrespective of age; and (2) older listeners show less benefit from dichotic presentation than younger listeners, and that this effect is evident in middle-age. The first hypothesis was clearly supported; across all 
listeners, detection thresholds were more acute in the dichotic conditions than in the monaural (or diotic) condition. The second hypothesis was also supported; the dichotic measurements - in particular the condition with antiphasic modulators presented to the two ears -differentiated successfully between the three age groups. In contrast, the monaural and diotic measurements were less able to differentiate mid-aged from older listeners. This suggests that dichotic FM detection is a more sensitive gauge of deficits in phase-locking ability across the age span than is monaural FM detection. In support of this, Moore et al. (2012b) found that sensitivity to binaural temporal fine structure cues in particular worsened with age even when absolute thresholds were not elevated. However, it must be acknowledged that this conclusion does not diminish support for the use of monaural lowrate FM detection as a sensitive gauge of declining auditory temporal processing in older listeners. Here, both monaural and diotic FM detection thresholds were able to differentiate the performance of the younger listeners from that of older listeners. This is in line with the conclusions of He et al. (2007), who found that older listeners had higher 5-Hz FM detection thresholds than did younger listeners. They also observed that - for the most part - this age effect was more pronounced in the $500-\mathrm{Hz}$ region than in the $4000-\mathrm{Hz}$ region, and interpreted this as pointing to a decline in phase-locking fidelity. It should be recognized that as low-rate FM detection worsens in monaural/diotic conditions the contribution of place cues to performance presumably becomes greater. If performance is limited by place cues, then it is unlikely that a dichotic stimulation mode will be beneficial.

The proposition that dichotic FM detection is to be preferred over monaural FM detection as a gauge of temporal fine structure processing raises the question of whether asymmetries in absolute sensitivity across ears would be problematic. Although subject inclusion criteria here required symmetric audiometric hearing at the test frequency, it is possible that this may not be typical in the general aging population. The study ofWitton et al. (2000) suggests that ear asymmetries are unlikely to be an issue, assuming that a difference in sensation level across the two ears is the only factor of importance underlying their pattern of results. They found that the magnitude of benefit brought about by adding a pure tone to the ear contralateral to the ear receiving the FM tone (i.e., Dichotic PT/FM) was relatively constant even when the level of the pure tone was reduced by about $50 \mathrm{~dB}$ below that of the FM tone. In order to determine whether a similar constancy applied here, all but two of the listeners participated in a supplementary condition in which one of the FM tones in the Dichotic FM/ $F M$ condition was reduced in level by $30 \mathrm{~dB}$ relative to the other tone, yielding presentation levels of 35 and $65 \mathrm{~dB}$ SPL respectively across ears. This resulted in a substantially reduced sensation level in one ear. The data for the symmetric and asymmetric DichoticFM/FM conditions are shown in Fig. 4. It can be seen that the level asymmetry uniformly increased detection thresholds for all age groups. This was confirmed with a repeated measures ANOVA that showed a significant effect of age group $(\mathrm{F}(1,31)=18.2 ; \mathrm{p}<0.01)$ and symmetry condition $(\mathrm{F}(1,31)=58.0, \mathrm{p}<0.01)$, and no interaction between these factors. It is not clear why the $30-\mathrm{dB}$ asymmetry resulted in poorer detection thresholds in this study but a similar asymmetry did not in the study of Witton et al. (2000). Perhaps this was due, in part, to the higher overall presentation level in that study (80 dB SPL). Another result of the level asymmetry effect observed here is that the elevation in dichotic FM detection threshold among the older listeners essentially removed any dichotic advantage relative to diotic listening. A repeated-measures ANOVA comparing the diotic thresholds with the asymmetric dichotic thresholds indicated a significant effect of age group $(\mathrm{F}(1,31)=22.2 ; \mathrm{p}$ $<0.01)$ and condition $(\mathrm{F}(1,31)=51.2 ; \mathrm{p}<0.01)$, and a significant interaction between these factors $(\mathrm{F}(2,31)=5.7 ; \mathrm{p}=0.008)$. Pair-wise comparisons indicated that, for both the younger and mid-aged groups, the thresholds for the asymmetric level remained significantly better than the diotic thresholds; however, for the older group there was no difference between the two sets of thresholds. Thus, in an older listener with a pronounced audiometric asymmetry, the benefit of dichotic FM detection could be compromised. 
However, it should be emphasized that the asymmetry in stimulation level tested here is not equivalent to an audiometric asymmetry and that these two types of asymmetries may have different effects on binaural temporal processing.

\section{Summary and Conclusion}

The detection of low-rate FM carried by a low-frequency tone has been interpreted as reflecting fidelity of temporal fine structure coding. This study examined whether this measure of temporal coding could be made more acute by using a dichotic FM detection task. On the one hand, dichotic FM acuity was clearly superior to monaural acuity irrespective of listener age. In addition, this measure differentiated between age groups more definitively than a diotic measure, especially in terms of distinguishing mid-aged from older listeners. On the other hand, older listeners showed relatively less benefit of dichotic listening, and their performance was weakly associated with their absolute sensitivity to the carrier. Finally, in the presence of a marked asymmetry in sensation level across ears, the older listeners showed no dichotic benefit. A parsimonious interpretation of the overall pattern of results, therefore, is that dichotic FM measurements have advantages over monaural measurements for the purposes of assessing age-related temporal processing effects, although a marked asymmetry in absolute thresholds across ears could undermine these advantages.

\section{Acknowledgments}

This work was supported by NIH NIDCD R01DC001507. The helpful comments of the Associate Editor, Brian C.J. Moore, and two anonymous reviewers are gratefully acknowledged. The manuscript also benefited from constructive discussions with Joseph W. Hall III and Emily Buss.

\section{References}

Batra R, Kuwada S, Fitzpatrick DC. Sensitivity to interaural temporal disparities of low- and highfrequency neurons in the superior olivary complex. II. Coincidence detection. J. Neurophysiol. 1997; 78:1237-1247. [PubMed: 9310415]

Buss E, Hall JW 3rd, Grose JH. Temporal fine-structure cues to speech and pure tone modulation in observers with sensorineural hearing loss. Ear. Hear. 2004; 25:242-250. [PubMed: 15179115]

Clinard CG, Tremblay KL, Krishnan AR. Aging alters the perception and physiological representation of frequency: evidence from human frequency- following response recordings. Hear. Res. 2010; 264:48-55. [PubMed: 19944140]

Dobreva MS, O'Neill WE, Paige GD. Influence of aging on human sound localization. J. Neurophysiol. 2011; 105:2471-2486. [PubMed: 21368004]

Ernst SM, Moore BC. The role of time and place cues in the detection of frequency modulation by hearing-impaired listeners. J. Acoust. Soc. Am. 2012; 131:4722-4731. [PubMed: 22712945]

Green GG, Heffer JS, Ross DA. The detectability of apparent source movement effected by interaural phase modulation [proceedings]. J. Physiol. 1976; 260:49P-50P.

Grose JH, Mamo SK. Processing of temporal fine structure as a function of age. Ear. Hear. 2010; 31:755-760. [PubMed: 20592614]

He NJ, Mills JH, Dubno JR. Frequency modulation detection: effects of age psychophysical method, and modulation waveform. J. Acoust .Soc. Am. 2007; 122:467-477. [PubMed: 17614504]

Hopkins K, Moore BCJ. Development of a fast method for measuring sensitivity to temporal fine structure information at low frequencies. Int.J. Audiol. 2010; 49:940-946. [PubMed: 20874427]

Hopkins K, Moore BCJ. The effects of age and cochlear hearing loss on temporal fine structure sensitivity, frequency selectivity, and speech reception in noise. J. Acoust. Soc. Am. 2011; 130:334-349. [PubMed: 21786903]

Lacher-Fougere S, Demany L. Modulation detection by normal and hearing-impaired listeners. Audiol. 1998; 37:109-121. 
Lacher-Fougere S, Demany L. Consequences of cochlear damage for the detection of interaural phase differences. J. Acoust .Soc. Am. 2005; 118:2519-2526. [PubMed: 16266172]

Langhans A, Kohlrausch A. Differences in auditory performance between monaural and dichotic conditions. I: masking thresholds in frozen noise. J. Acoust. Soc. Am. 1992; 91:3456-3470. [PubMed: 1619122]

Moore BCJ, Sek A. Detection of frequency modulation at low modulation rates: evidence for a mechanism based on phase locking. J. Acoust. Soc. Am. 1996; 100:2320-2331. [PubMed: 8865639]

Moore BCJ, Skrodzka E. Detection of frequency modulation by hearing- impaired listeners: effects of carrier frequency, modulation rate, and added amplitude modulation. J. Acoust. Soc. Am. 2002; 111:327-335. [PubMed: 11833538]

Moore BCJ, Glasberg BR, Stoev M, Fullgrabe C, Hopkins K. The influence of age and high-frequency hearing loss on sensitivity to temporal fine structure at low frequencies (L). J. Acoust. Soc. Am. 2012a; 131:1003-1006. [PubMed: 22352474]

Moore BCJ, Vickers DA, Mehta A. The effects of age on temporal fine structure sensitivity in monaural and binaural conditions. Int J Audiol. 2012b [in press].

Ross B, Fujioka T, Tremblay KL, Picton TW. Aging in binaural hearing begins in mid-life: evidence from cortical auditory-evoked responses to changes in interaural phase. J. Neurosci. 2007; 27:11172-11178. [PubMed: 17942712]

Strelcyk O, Dau T. Relations between frequency selectivity, temporal fine- structure processing, and speech reception in impaired hearing. J. Acoust. Soc. Am. 2009; 125:3328-3345. [PubMed: 19425674]

Witton C, Green GG, Rees A, Henning GB. Monaural and binaural detection of sinusoidal phase modulation of a 500-Hz tone. J. Acoust. Soc. Am. 2000; 108:1826-1833. [PubMed: 11051509] 


\begin{tabular}{|c|c|}
\hline • & $\begin{array}{l}\text { Diotic FM detection was superior to monaural detection irrespective of } \\
\text { listener age }\end{array}$ \\
\hline • & $\begin{array}{l}\text { Dichotic FM detection was superior to diotic detection irrespective of listener } \\
\text { age }\end{array}$ \\
\hline • & $\begin{array}{l}\text { Dichotic FM performance differentiated between younger, mid-aged, and } \\
\text { older listeners }\end{array}$ \\
\hline • & $\begin{array}{l}\text { Older listeners did not exhibit dichotic benefit when the presentation levels } \\
\text { were markedly asymmetric }\end{array}$ \\
\hline
\end{tabular}




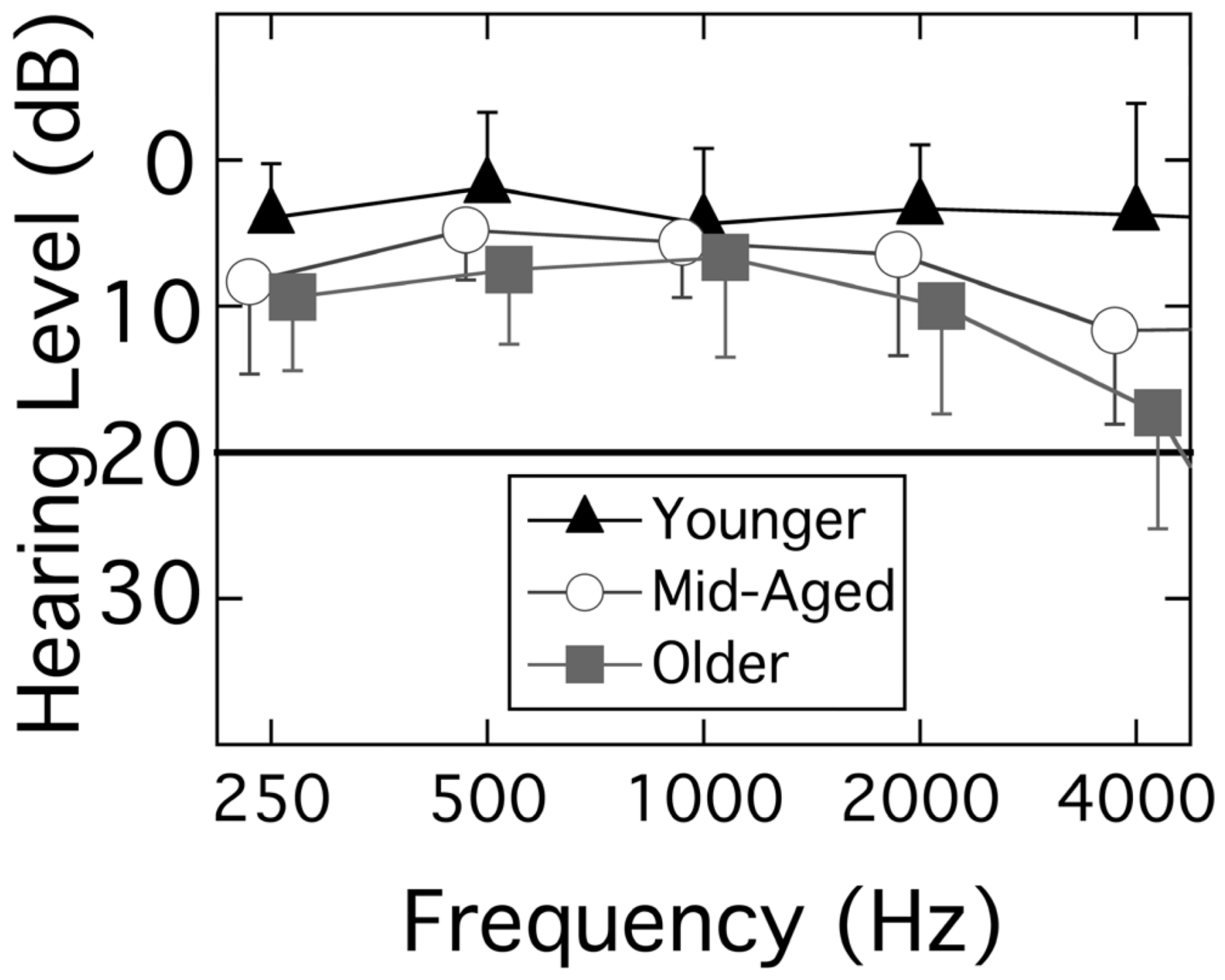

Fig. 1.

Mean audiometric thresholds in $\mathrm{dB}$ HL for the octave frequencies $250-4000 \mathrm{~Hz}$ for the younger (triangles), mid-aged (circles), and older (squares) groups. Error bars show 1 standard deviation. 


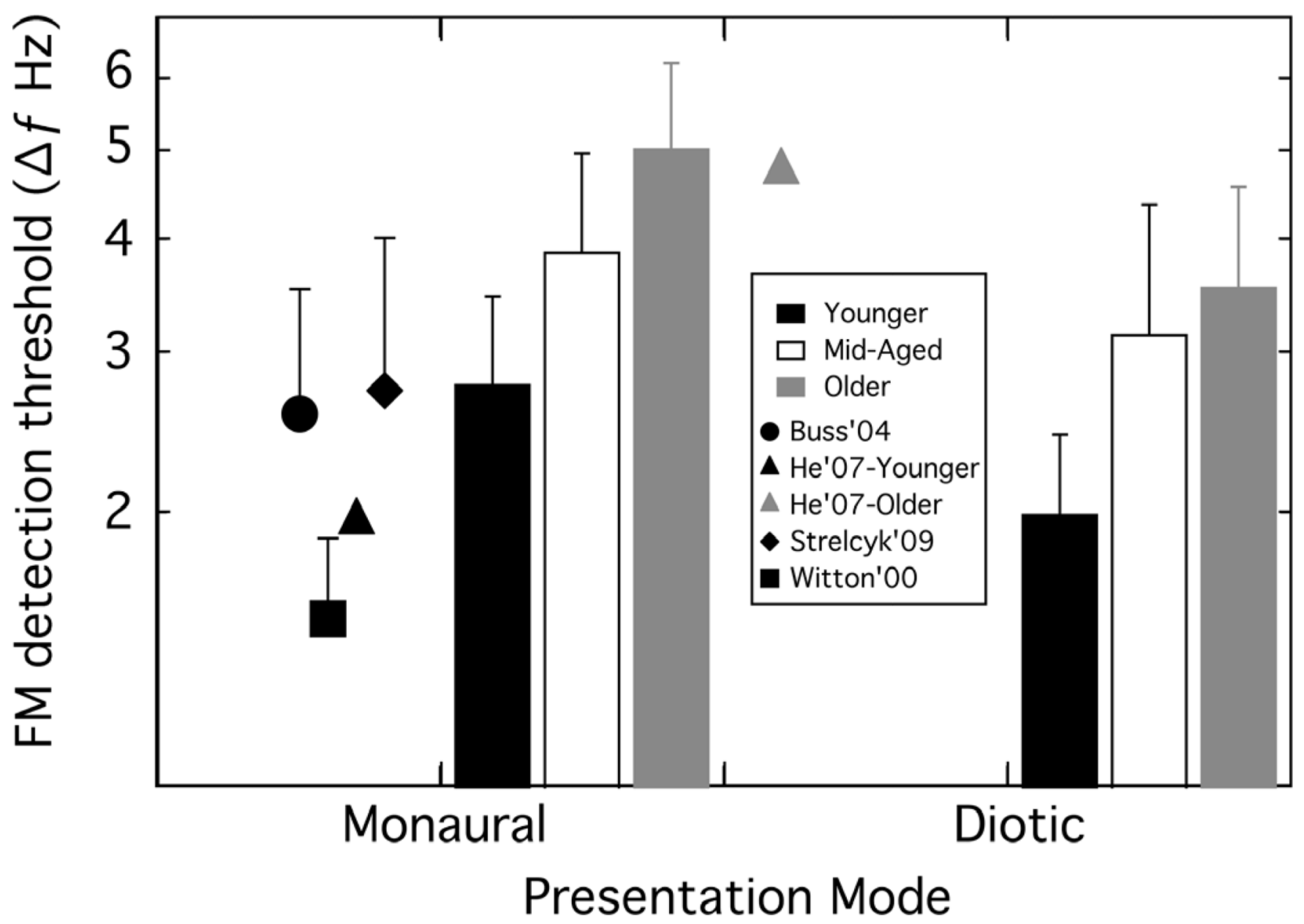

Fig. 2.

FM detection thresholds for monaural and diotic conditions. Bar shading indicates age group as shown by the key. Error bars show 1 standard deviation. Additional data from other studies include:Buss et al. (2004) [circle]; Witton et al. (2000) [square];He et al. (2007) [triangles: black (young); gray (older)]; and Strelcyk et al. (2009) (diamond). 


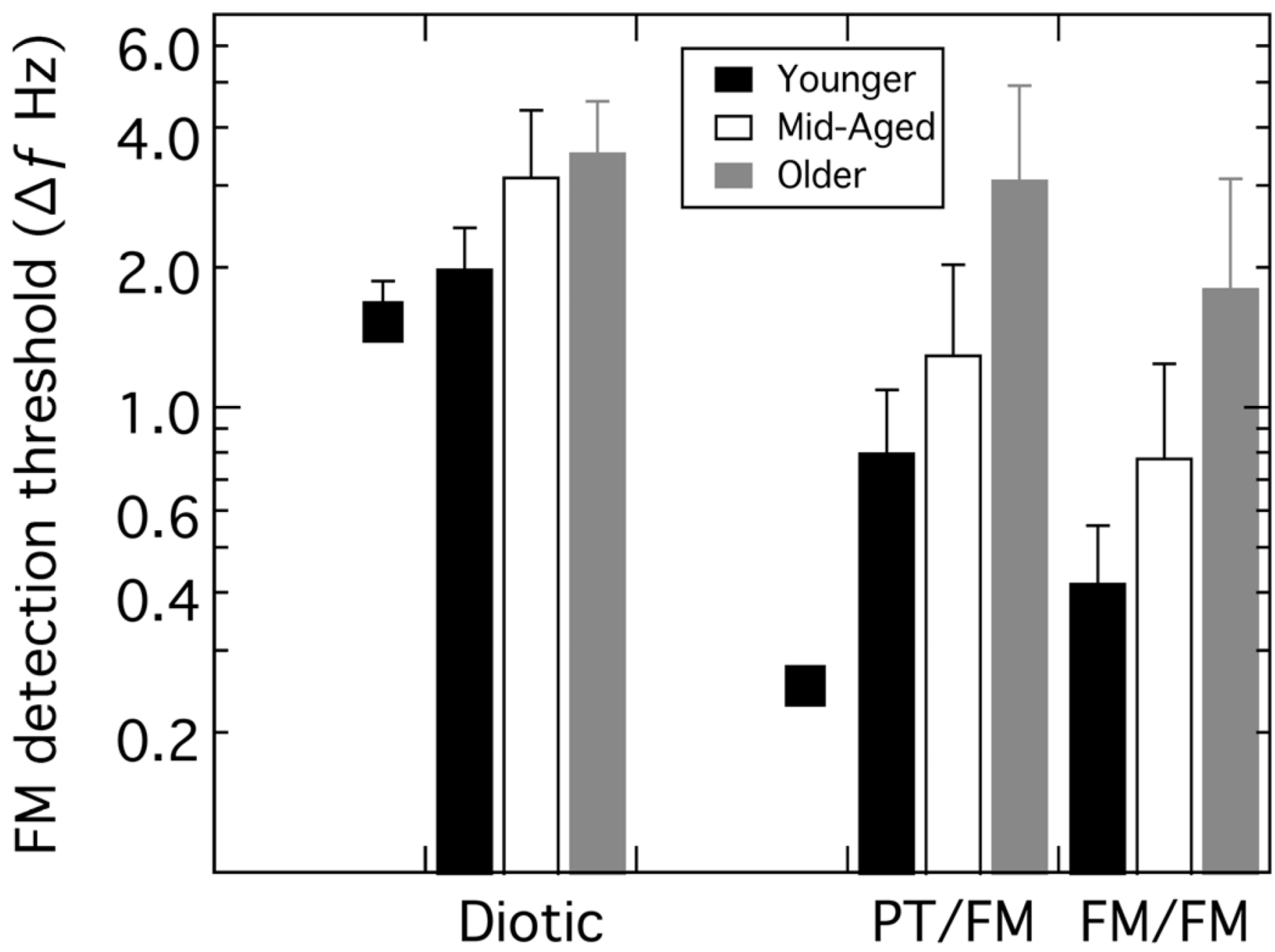

\section{Condition}

Fig. 3.

FM detection thresholds for the three binaural conditions: Diotic, Dichotic PT/FM, and Dichotic FM/FM. Bar shading indicates age group as shown by the key. Error bars show 1 standard deviation. Filled squares show data from Witton et al. (2000). 


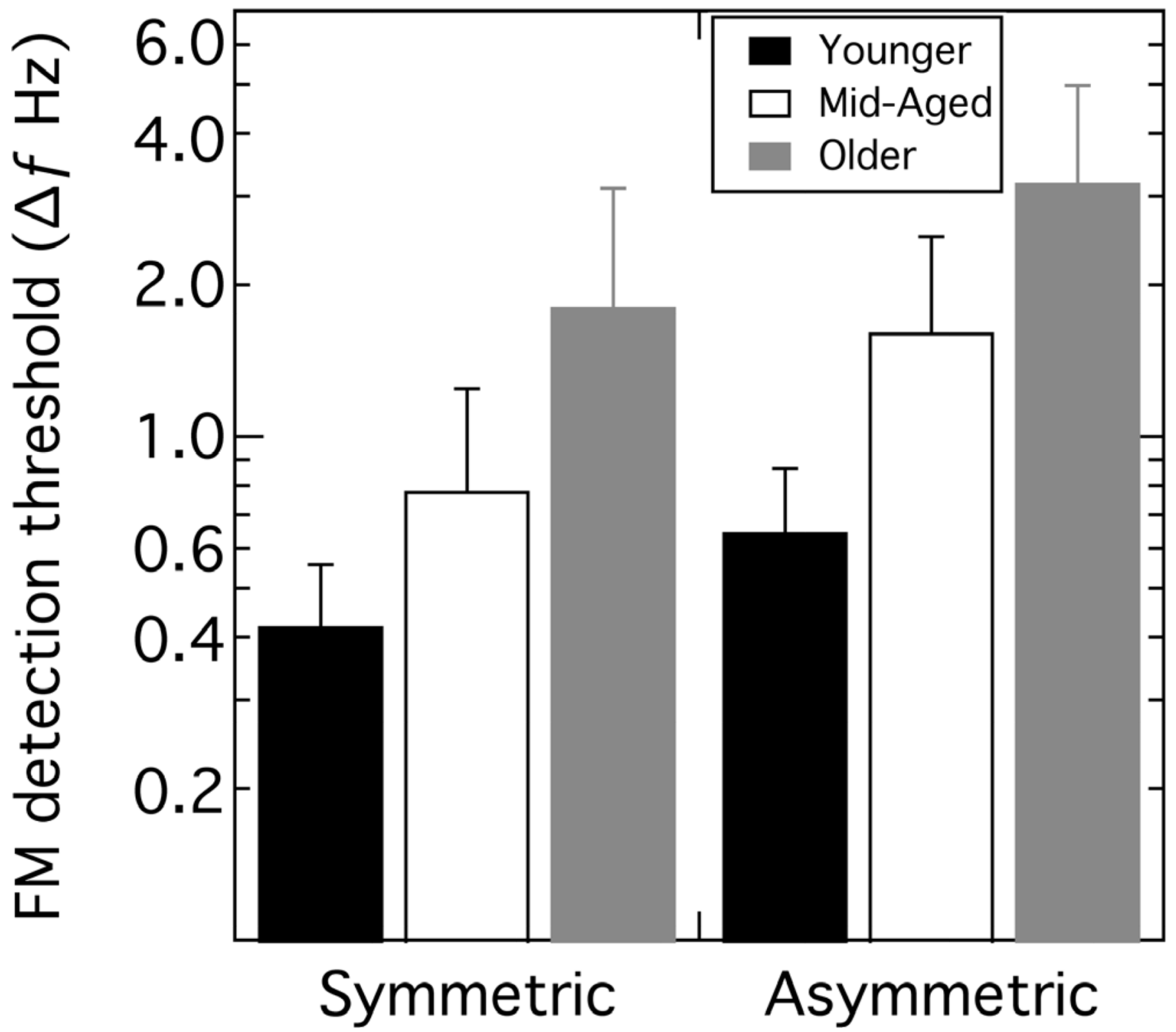

Fig. 4.

FM detection thresholds for symmetric and asymmetric presentation levels. Bar shading indicates age group as shown by the key. Error bars show 1 standard deviation. 